\title{
Right prefrontalTMS disrupts interregional anticipatory EEG alpha activity during shifting of visuospatial attention
}

\author{
Paul Sauseng ${ }^{1,2}$ *, Jan F. Feldheim ${ }^{2}$, Roman Freunberger ${ }^{3,4}$ and Friedhelm C. Hummel ${ }^{2}$ \\ 1 Department of Psychology, University of Surrey, Guildford, UK \\ 2 Brain Imaging and Neurostimulation Laboratory, Department of Neurology, Universitätsklinikum Hamburg-Eppendorf, Hamburg, Germany \\ ${ }^{3}$ Department of Psychology, University of Salzburg, Salzburg, Austria \\ ${ }^{4}$ Center for Lifespan Psychology, Max Planck Institute for Human Development, Berlin, Germany
}

\section{Edited by:}

Gregor Thut, University of Glasgow, UK

\section{Reviewed by:}

Valentin Wyart, University of Oxford, UK

Paul Taylor, Ludwig Maximilian University of Munich, Germany

\section{*Correspondence:}

Paul Sauseng, Department of Psychology, University of Surrey, Guildford, Surrey, GU2 7XH, UK. e-mail: p.sauseng@surrey.ac.uk
Visual attention can be shifted in space without moving the eyes. Amplitude decrease of rhythmical brain activity around $10 \mathrm{~Hz}$ (so called alpha activity) at contralateral posterior sites has been reported during covered shifts of visuospatial attention to one visual hemi-field. Alpha amplitude increase, on the other hand, can be found at ipsilateral visual cortex. There is some evidence suggesting an involvement of prefrontal brain areas during the control of attention-related anticipatory alpha amplitude asymmetry. This open question has been studied in detail using a multimodal approach combining transcranial magnetic stimulation (TMS) and multichannel electroencephalography (EEG) in healthy humans. Slow $(1 \mathrm{~Hz})$ repetitive TMS leading to reduced excitability of the stimulation site was delivered either to right frontal eye field (FEF) or a control site (vertex). Subsequently, participants had to perform a spatial cuing task in which covert shifts of attention were required to either the left or the right visual hemi-field. After stimulation at the vertex (control condition) a pattern of anticipatory, attention-related ipsilateral alpha increase/contralateral alpha decrease over posterior recording sites could be obtained. Additionally, there was pronounced coupling between (in particular right) FEF and posterior brain sites at EEG alpha frequency. When, however, right prefrontal cortex had been virtually lesioned preceding the task, these EEG correlates of visuospatial attention were attenuated. Notably, the effect of TMS at the right FEF on interregional fronto-parietal alpha coupling predicted the effect of TMS on response times. This suggests that visual attention processes associated with posterior EEG alpha activity are at least partly top-down controlled by the prefrontal cortex.

Keywords: frontal eye field, fronto-parietal attention network, repetitive transcranial magnetic stimulation, top-down control

\section{INTRODUCTION}

Neuroimaging studies suggest that in humans visuospatial attention is controlled by a distributed fronto-parietal cortical network (for an overview see Corbetta and Shulman, 2002). It has been suggested that in particular in the right hemisphere a prefrontal cortical region known as the frontal eye field (FEF) together with posterior cortical areas around the intraparietal sulcus (IPS) form a dorsal attention network subserving top-down control of visual attention. Originally, the FEF has been associated with motor control of saccadic eye movements. However, there is strong evidence coming from electrophysiological studies in monkeys suggesting that the FEF is also involved in covered shifting of visual attention in space, i.e., directing the focus of visual attention to parts of the visual field without performing eye movements (Moore and Fallah, 2001). Moreover, electrical stimulation of the FEF was shown to result in increased firing rates of visual area V4 in monkeys (Moore and Armstrong, 2003). This suggests that the FEF and higher visual areas are functionally linked during the control of visual attention. Evidence that the FEF is causally linked to the control of attention also in humans comes from recent studies using transcranial magnetic stimulation (TMS) to transiently disrupt neural processing in this brain region while participants are engaged with performing on attention tasks (see e.g., Grosbras and Paus, 2002; Taylor et al., 2007; Smith et al., 2009). Notably, Taylor et al. (2007) investigated the influence of TMS applied to the FEF on neural activity at posterior brain sites. They combined repetitive TMS (rTMS) with electroencephalographic (EEG) recordings. In an attentional cuing task the authors delivered rTMS to the FEF in the time interval between a central cue and a peripheral target. Taylor et al. (2007) found that compared to a control condition event-related potential (ERP) components elicited by targets and associated with visuospatial attention were altered at parieto-occipital recording sites. These findings are well in line with Moore and Armstrong's (2003) observations in monkeys and suggest that also in humans the FEF has a significant impact on posterior attention-related neural activity.

Looking at electrophysiological recordings, directing visuospatial attention leads to far less subtle effects in posterior brain areas than in the FEF. There are prominent attention-related modulations of early ERP components reported (for review see Hillyard and Anllo-Vento, 1998) which can be localized to extrastriate cortex (Di Russo et al., 2003). These findings suggest that the neural response to visual stimuli in posterior brain areas is amplified when the target is presented at an attended location in the visual 
field. However, at parietal recording sites EEG correlates of shifting visuospatial attention can already be obtained prior to target presentation but in a delay interval between cue and target. Modulation of rhythmical posterior EEG activity has been shown to correlate with visual attention. It has consistently been demonstrated that the amplitude of posterior alpha oscillations (around $10 \mathrm{~Hz}$ ) is attenuated contralaterally to an attended part of the visual field, whereas there is usually increased alpha activity at posterior recording sites ipsilateral to the attended visual field (Worden et al., 2000; Sauseng et al., 2005a; Thut et al., 2006; Rihs et al., 2007, 2009; Freunberger et al., 2008). Recently, Romei et al. (2010) were able to show that entraining this attention-related pattern of alpha activity via rhythmical TMS can modulate visuo-perceptual performance indicating that contralateral EEG alpha amplitude decrease and/or ipsilateral alpha amplitude increase play a crucial role during shifting of visuospatial attention.

However, it is still not completely understood if and how posterior attention-related EEG alpha amplitude modulation is influenced by the prefrontal cortex. There is certain evidence that this actually might be the case: (i) Research by Taylor et al. (2007), as mentioned above, indicates that disruption of the FEF leads to alteration of post-target ERP components. (ii) Functional magnetic resonance imaging ( $\mathrm{fMRI}$ ) investigating causal relationships between frontal and posterior brain areas suggests a top-down impact of the FEF onto IPS (Bressler et al., 2008). And (iii) for theta oscillations initial upstream activation from posterior to frontal areas and a later shift back from prefrontal to posterior cortical regions during direction of attention has been shown (Green and McDonald, 2008). The question how this fronto-parietal interaction contributes to attention-related findings at alpha frequency, however, has remained open. Recently, Capotosto et al. (2009) conducted a study in which brief rTMS trains where delivered either to the right FEF, IPS, or control sites during the cue-target interval of a visuospatial attention task. EEG alpha amplitude was investigated for the pre-target time window following rTMS. Performance on the task was attenuated after FEF and most effectively after IPS stimulation. More importantly, Capotosto et al. (2009) found less lateralized attention-related alpha activity after FEF and IPS being virtually lesioned compared to control rTMS. The effects following FEF stimulation, however, were rather moderate while there were strong effects of rTMS applied over the IPS. Nevertheless, these data provide good evidence supporting the view that FEF is involved in control of attention-related EEG alpha at posterior sites. However, so far it is not completely clear how this prefrontal control over posterior alpha oscillations is implemented in the brain. Sauseng et al. (2005a) presented results suggesting that during covert shifts of visual attention interregional phase synchronization between prefrontal and parietal electrode sites was obtained. Moreover, EEG results from a visual task requiring high level of top-down processing indicate posterior alpha amplitude being controlled by prefrontal cortex and that this control might be achieved by interregional coupling at the alpha frequency range (Sauseng et al., 2005b). The important question addressed here is whether a virtual lesion to the FEF will lead to altered patterns of interregional connectivity between anterior and posterior brain regions. It is also investigated whether such modulated connectivity patterns are responsible for remote effects from magnetic stimulation at the FEF on attention-related posterior alpha amplitude.

Here we ran a visuospatial attention task immediately after lowfrequency rTMS either to the right FEF or the vertex (as a control site). TMS was applied to decrease neural activity of the stimulated brain region. Within the applied TMS protocol induced effects were outlasting the stimulation for at least the time period the visuospatial attention task was carried out by the subjects. During the task EEG was recorded to investigate the role of interregional functional coupling between FEF and posterior parietal areas during the control of parietal attention-related alpha activity. We hypothesized that rTMS over the FEF would lead to (i) attenuated attention-related lateralized alpha activity at posterior recording sites, possibly caused by (ii) decreased fronto-parietal connectivity in the alpha frequency range.

\section{MATERIALS AND METHODS PARTICIPANTS}

Twelve volunteers (four males) with a mean age of 27.2 years $(\mathrm{SD}=4.1)$ participated in the study. Except one subject all participants were right handed. In one subject EEG was strongly contaminated with eye blink artifacts. Therefore this subject had to be excluded from EEG analysis. However, their behavioral data was used in the current analysis. All subjects gave written informed consent, and the experiment was carried out in agreement with the Declaration of Helsinki.

\section{EXPERIMENTAL PARADIGM}

A visuospatial cued attention task was run. Participants sat in front of a computer screen with their gaze fixated to a black cross presented on gray background. The fixation cross was shown $5.5^{\circ}$ visual angle above the center of the screen. In each trial an acoustic cue was presented, either a tone at 500 or $1000 \mathrm{~Hz}$. Acoustic cue stimuli were presented with loudspeakers positioned left and right to the PC screen. Half of the subjects were instructed to covertly (without moving their gaze away from the fixation cross) shift their visual attention to the left visual hemi-field when a $500-\mathrm{Hz}$ tone and to the right when a cue with $1000 \mathrm{~Hz}$ had been presented. The other half of subjects had to attend to the left visual field after a $1000-\mathrm{Hz}$ cue and to the right after a tone with $500 \mathrm{~Hz}$. After a jittered interval of 600-800 ms after cue presentation a visual target was presented at the screen for $83 \mathrm{~ms}$. The target either was a "p" or a " $q$ " ( $1.5^{\circ}$ visual angle $)$ and was either presented $12.7^{\circ}$ visual angle to the left or to the right of the screen's center. We chose to use an acoustic cue instead of a visual one (e.g., an arrow) in order not to mask effects of shifting visual attention in the cue-target interval by visual processing of the cue. In total the experiment consisted of 400 trials of which in half attention had to be directed to the left and in half to the right visual hemi-field. In 75\% of the trials target location was congruent with the cued visual hemi-field (valid trials). The participants' task was to indicate as fast and accurately as possible whether the target was a "p" or a "q." They did so by pressing one of two buttons with their index finger of the dominant hand. Between trials there was an inter-trial interval jittering between 2000 and 3000 ms. Auditory and visual stimulation were controlled by Presentation ${ }^{\circledR} 0.71$ software (NeuroBehavioralSystems). Before carrying out the experiment participants performed 
a training block consisting of 50 trials to get familiarized to the task.

\section{EEG RECORDINGS}

Using a BrainProducts ${ }^{\circledR}$ BrainAmpMR+ amplifier EEG was recorded from 31 sites. $\mathrm{Ag}-\mathrm{AgCl}$ electrodes were positioned according the extended 10-20-system using an EasyCap ${ }^{\circledR}$ from which adaptors were removed around the TMS stimulation site and right primary motor cortex to reduce the distance between TMS coil and scalp. Electrodes close to the TMS coil were carefully taped to avoid direct contact between the coil and EEG electrodes. Recording reference was set to the tip of the nose with a grounding electrode being placed to the forehead, and electrooculogram was recorded with an additional channel to control for horizontal eye movements. Data were recorded with a sampling rate of $1000 \mathrm{~Hz}$. A notch filter at $50 \mathrm{~Hz}$ was applied and data were acquired between 0.016 and $70 \mathrm{~Hz}$. Impedance was kept below $5 \mathrm{k} \Omega$.

\section{RTMS PROTOCOL}

A Magstim ${ }^{\circledR}$ Rapid2 stimulator with a 70-mm figure-eight coil was used. rTMS at a rate of $1 \mathrm{~Hz}$ was delivered either to the right FEF or the vertex (electrode site $\mathrm{Cz}$ ) for $15 \mathrm{~min}$ (900 pulses). This stimulation protocol leads to LTD-like effects and a reduction of neural activity outlasting the stimulation up to $30 \mathrm{~min}$ (Chen et al., 1997). Every participant underwent stimulation twice, once at FEF and once at the vertex. The two sessions were separated by at least 1 day, and the order of conditions was balanced between subjects. Stimulation site for right hemispheric FEF was determined according to O'Shea et al. (2004). The cortical representation of the left hand's FDI muscle was used as an anchor point from which FEF stimulation site was rostral by 3 and $5 \mathrm{~cm}$ lateral from the sagittal midline. For FEF stimulation the coil was positioned with the handle perpendicular to the sagittal midline (O'Shea et al., 2004). For rTMS at the vertex the coil was held with the handle pointing backward parallel to the sagittal midline. As stimulation intensity $110 \%$ of resting motor threshold determined according to Rossini et al. (1994) was used. This stimulation protocol was well in line with published safety guidelines (Wassermann, 1998; Rossi et al., 2009).

\section{EXPERIMENTAL PROCEDURE}

After determination of stimulation sites and stimulation intensity participants performed the training block on the visuospatial attention task. Thereafter, they received rTMS either over the right FEF or the vertex. Immediately after the 15-min of rTMS EEG recording was started and participants performed the cued attention task with a duration of $21 \mathrm{~min}$.

\section{BEHAVIORAL DATA ANALYSIS}

On each trial subjects had to respond as fast and as correctly as possible indicating whether the target was a "p" or a "q." Average response times and percent accurate responses were analyzed for each stimulation condition, attention directed to the left or right, as well as valid and invalid conditions separately.

\section{EEG DATA ANALYSIS}

EEG data analysis was carried out using BrainVision 2 (BrainProducts ${ }^{\circledR}$ ) and Matlab ${ }^{\circledR} 7.0$ (MathWorks) software. After a highpass filter at $0.5 \mathrm{~Hz}$ Laplacian current source density (CSD) transformation was applied to attenuate effects of volume conduction on local amplitude and interregional functional coupling. Next, trials containing artifacts, e.g., due to eye movements, blinks, muscle activity, etc., were removed after manual visual data inspection. Trials exhibiting horizontal EOG activity, i.e., trials in which participants did not fixate the cross on the computer screen, were rejected as well. Data were segmented into intervals of $500 \mathrm{~ms}$ immediately preceding target presentation separately for attention to be shifted to the right and attention directed to the left visual hemi-field. Then Fast Fourier Transformation was applied and trials were averaged in frequency domain for the two conditions separately. This was done for EEG after FEF stimulation as well as after rTMS over the vertex. Spectral amplitude between 10 and $12 \mathrm{~Hz}$ at parietal and occipital recording sites ( $\mathrm{P} 7, \mathrm{P} 3, \mathrm{PO} 3, \mathrm{O} 1, \mathrm{P} 8, \mathrm{P} 4, \mathrm{PO} 4$, O2) was used for statistical analysis since findings by Sauseng et al. (2005a,b) suggest fronto-parietal connectivity at this frequency range to play an important role in top-down processing of visuospatial information. Capotosto et al. (2009) used individual alpha frequency peaks for adjusting upper alpha frequency bands. In the present study all subjects exhibited a resting individual alpha peak frequency (at posterior recording sites) between 10 and $10.5 \mathrm{~Hz}$ (mean individual alpha frequency $=10.31, \mathrm{SD}=0.26$ ). Since frequency resolution of amplitude spectra in the experiment was at $2 \mathrm{~Hz}$ and in order to keep filter properties identical for all subjects we did not adjust frequency bands individually.

For interregional connectivity analysis CSD transformed and artifact free data were submitted to band-pass filtering between 10 and $12 \mathrm{~Hz}$ (Butterworth filter; roll-off at $48 \mathrm{~dB} / \mathrm{Oct}$ ). Data were segmented as above. Cross-correlations of filtered segments between prefrontal recording sites being close to estimated sites of the FEF (FC3 and FC4) and posterior recording sites (P7, P3, PO3, $\mathrm{O} 1, \mathrm{P} 8, \mathrm{P} 4, \mathrm{PO} 4, \mathrm{O} 2)$ were calculated on a single-trial basis. Thus, for each single-trial filtered EEG signals from one prefrontal and one posterior site were correlated, and then this procedure was repeated again and again with the signal from one recording site shifted by always one sample point in respect to the signal from the second recording site. This was done in two directions. As a result a cross-correlogram was obtained for each single-trial displaying correlation coefficients as a function of latency shift between the two signals. Then cross-correlograms were averaged over trials separately for each of the 16 electrode pairs, FEF vs. vertex stimulation and attention directed to the left vs. right visual hemi-field. These averaged cross-correlograms indicate how well the signals from two recording sites correspond either with a latency difference of $0 \mathrm{~ms}$ or a certain latency delay. If information is processed sequentially in brain regions lying under two different recording sites one would expect that a similar EEG signal should be obtained from the two sites. However, due to sequential processing (and neural transmission time from one to the other site) highest correlations are not expected at a latency shift of $0 \mathrm{~ms}$ but with a slight lag in either direction (indicating which of the two sites is leading and which is trailing). In order to find out whether there was a consistent latency shift of upper alpha activity from prefrontal to posterior recording sites indicating top-down activation from prefrontal cortex on posterior brain areas, for each subject separately latencies of maximal correlation coefficient in the trial-averaged cross-correlogram were determined. This approach 
allows determining whether frontal sites are leading or trailing posterior sites (for more detail on this method see Sauseng et al., 2004, 2005b). In addition the value of maximal correlation coefficient was used in each subject, condition, and electrode pair for later statistical analysis.

\section{RESULTS \\ BEHAVIORAL RESULTS}

Using mean response times a three-way repeated measures ANOVA with factors STIMULATION (FEF, vertex), CUE VALIDITY (valid, invalid), and VISUAL HEMI-FIELD (left attended, right attended) was run. There was a significant main effect for factor CUE VALIDITY $\left(F_{1 / 11}=8.46, p=0.014\right)$, indicating an average (rTMS independent) response time advantage for valid compared to invalid trials by $51.7 \mathrm{~ms}$. The interaction between factors STIMULATION and CUE VALIDITY was marginally significant $\left(F_{1 / 11}=3.93, p=0.073\right)$. Pair-wise comparisons between rTMS over FEF vs. vertex (paired-sample $t$-tests, corrected for multiple comparison according to FDR correction as suggested by Benjamini and Hochberg, 1995) indicate slower response times after FEF stimulation for valid trials only (attention left: $t_{11}=2.94$, $p<0.05$ corrected; attention right: $t_{11}=3.25, p<0.05$ corrected; Figure 1). There was no such effect found in invalid trials. Additionally, there was only a significant validity effect (faster response times for valid compared to invalid trials) after stimulation at the vertex (attention left: $t_{11}=2.75, p<0.05$ corrected for multiple comparisons according to Benjamini and Hochberg, 1995; attention right: $t_{11}=3.56, p<0.05$ corrected; Figure 1$)$. No such effect was obtained after rTMS over the right FEF. In terms of response accuracy participants performed close to ceiling. There were no effects found for accuracy on neither type of rTMS, cue validity nor on visual hemi-field to which attention had to be shifted.

\section{POSTERIOR UPPER ALPHA AMPLITUDE}

Upper alpha $(10-12 \mathrm{~Hz})$ amplitude values of posterior recording sites (P7, P3, P4, P8, P03, PO4, O1, and O2) were submitted to

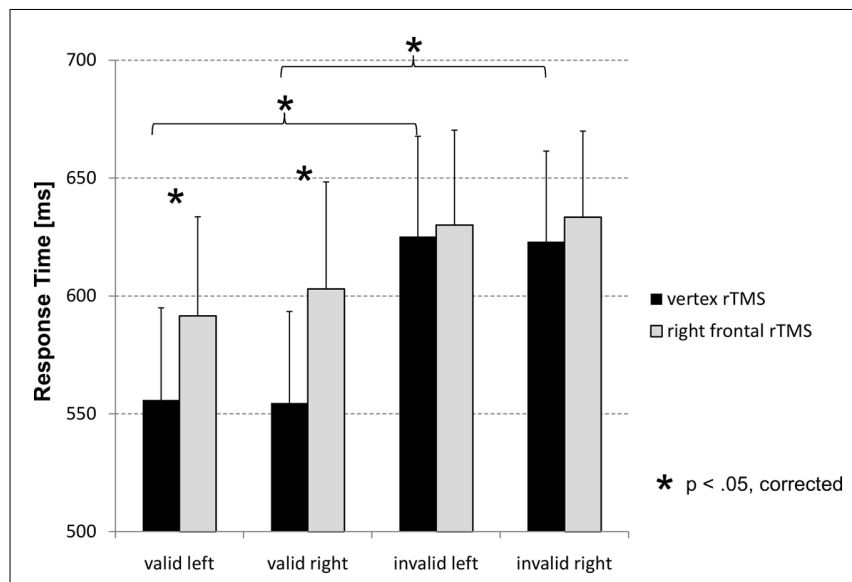

FIGURE 1 | Mean response times as a function of experimental and stimulation condition. Only in valid trials response times were delayed after rTMS at the right FEF compared to stimulation at the vertex (control site). This was the case for trials with the left visual hemi-field attended as well as when attention had been shifted toward the right visual hemi-field. a repeated measures ANOVA with factors STIMULATION (FEF, vertex), VISUAL HEMI-FIELD (left attended, right attended), HEMISPHERE (left, right), and RECORDING SITE (inferior parietal [P7 and P8], dorsal parietal [P3 and P4], parieto-occipital [PO3 and PO4], occipital [O1 and O2]). Greenhouse-Geisser correction was applied where required. There was a significant main effect for factor RECORDING SITE $\left(F_{3 / 30}=7.42, p=0.008\right)$. A significant interaction between factors VISUAL HEMI-FIELD and HEMISPHERE $\left(F_{1 / 10}=16.30, p=0.002\right)$ indicates higher upper alpha amplitude in the hemisphere ipsilateral to the attended visual hemi-field than contralateral. Most importantly however, the interaction between factors STIMULATION, VISUAL HEMIFIELD, and HEMISPHERE $\left(F_{1 / 10}=5.72, p=0.038\right)$ and the four-way interaction $\left(F_{3 / 30}=6.25, p=0.003\right)$ were significant. As depicted in Figure 2 the common pattern of stronger ipsilateral alpha activity compared to amplitude contralateral to the attended hemi-field as a signature of shifting visuospatial attention has been obtained after rTMS over the vertex (control site) only. There is no such effect elicited after rTMS over the right FEF. Post hoc $t$ tests comparing trials with the left vs. the right visual hemi-field attended indicate significant differences for electrode sites P3, PO3, and $\mathrm{O} 1\left(t_{10}>3.34, p<0.01\right.$ corrected for multiple comparisons according to Benjamini and Hochberg, 1995) after vertex rTMS. No significant effects were obtained for the right hemisphere or after FEF stimulation.

\section{INTERREGIONAL FUNCTIONAL COUPLING}

For each participant and electrode pair latencies of the averaged cross-correlogram's maximum were used as indicator for latency shifts of upper alpha activity and their direction. To evaluate consistency of latency shifts over the sample of subjects one sample $t$-tests were run for each of the 16 electrode pairs, each of the experimental conditions (attention shifted to left, attention shifted to right) and each of the two stimulation conditions separately. Results were corrected for multiple comparisons using FDR correction as suggested by Benjamini and Hochberg (1995). As can be seen in Figure 3 in general posterior recording sites always trailed prefrontal sites suggesting top-down influence of prefrontal brain areas to posterior cortical regions. Latency shifts from frontal to posterior sites were between 16.5 and $34.0 \mathrm{~ms}$. This is a range similar to previously reported long-range latency shifts at EEG alpha frequency and physiologically plausible (Schack et al., 2003). After rTMS at the control site there were consistent upper alpha latency shifts from electrode site FC4 to P3 $(p<0.01)$, PO3 $(p<0.05)$, $\mathrm{P} 7(p<0.05)$, and $\mathrm{O} 1(p<0.05)$ when attention was directed to the left visual hemi-field. When the right visual hemi-field was attended there were significant latency shifts from recording site FC3 to P7 $(p<0.01)$ and P3 $(p<0.01)$ as well as from FC4 to P3 $(p<0.01)$, P7 $(p<0.01)$, PO3 $(p<0.01)$, and PO4 $(p<0.05$; all $p$-values corrected for multiple comparison). After rTMS at the right FEF this connectivity pattern was changed dramatically. As can be seen in Figure 3 only three electrode pairs involving electrode site FC4 (the one closest to stimulation site) remained showing significant latency shifts from frontal to posterior (FC4 to P7 $(p<0.05)$, P3 $(p<0.05)$, and PO3 $(p<0.01)$ when attention was shifted to the right). In addition, there were significant latency shifts from FC3 to P7 $(p<0.05)$ when the left visual hemi-field was 

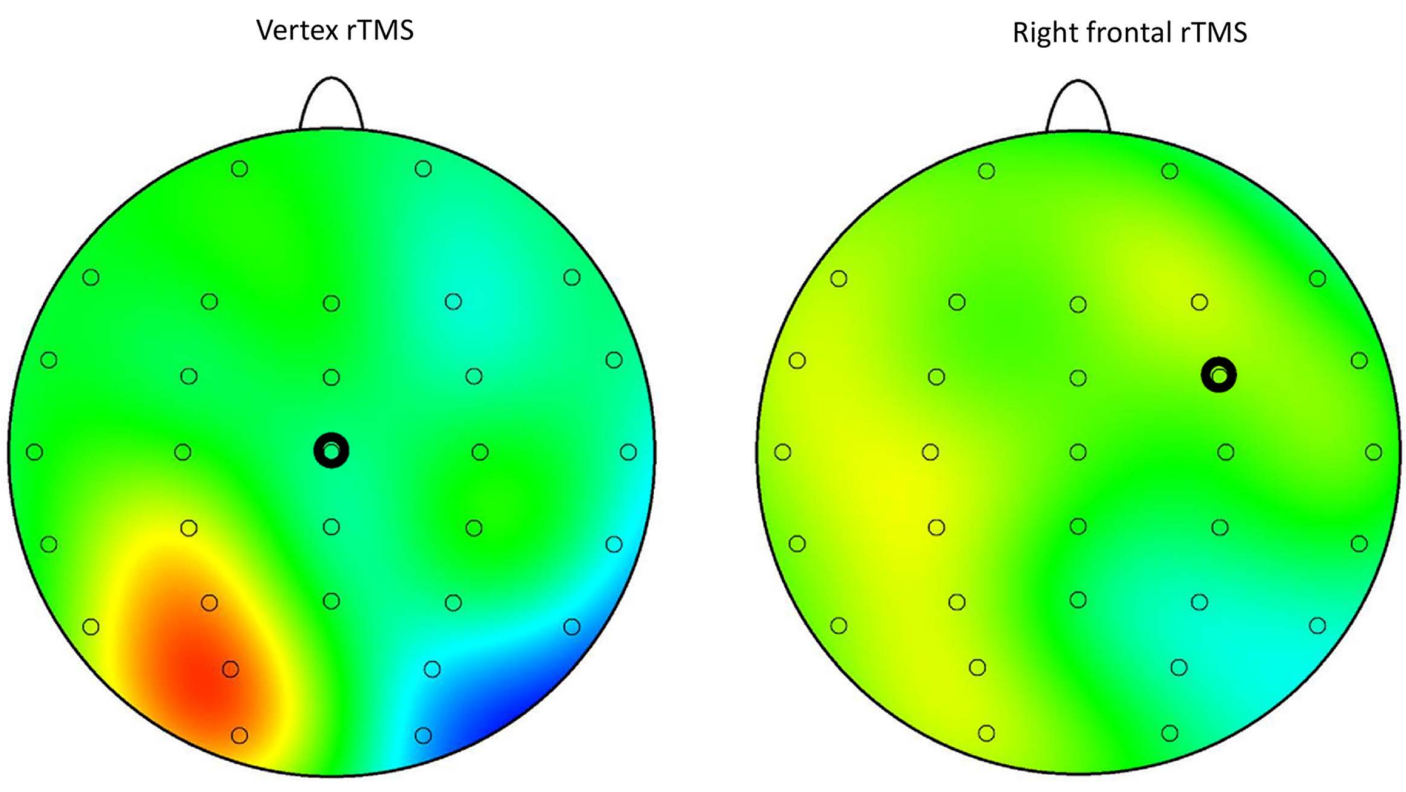

- approximate stimulation site

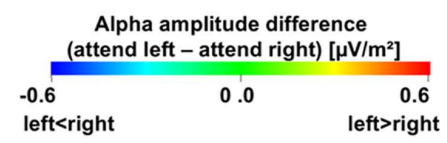

FIGURE 2 | Lateralized attention-related alpha amplitude.

Topographical maps display anticipatory upper alpha amplitude difference values between conditions with attention directed to the left minus right visual hemi-field. Since ipsilateral alpha amplitude is increased compared to contralateral activity these difference values (attention left minus attention right) are positive for electrode sites over the left hemisphere, and they are negative for recording sites at the right hemisphere. Positive difference values are coded by warm, negative values by cold colors. Note that increased ipsilateral upper alpha amplitude at posterior recording sites as correlate of shifting visuospatial attention is only obtained after rTMS delivered at the control site. This pattern is absent after setting a virtual lesion at the right FEF. attended. For all electrode pairs showing an effect with $p<0.01$ the $t$-value was $>3.26$, for all exhibiting an effect on the corrected $5 \%$ significance level $t$-values were $>2.43(\mathrm{df}=10)$.

As a measure of coupling strength between prefrontal and posterior sites maximal cross-correlation coefficient was used. A repeated measures ANOVA with factors STIMULATION (FEF, vertex), VISUAL HEMI-FIELD (left attended, right attended), PREFRONTAL SITE (FC3, FC4), POSTERIOR HEMISPHERE (left, right), and POSTERIOR RECORDING SITE (inferior parietal [P7 and P8], dorsal parietal [P3 and P4], parieto-occipital [PO3 and $\mathrm{PO} 4]$, occipital [O1 and O2]) was run. A significant interaction between factors STIMULATION and PREFRONTAL SITE $\left(F_{1 / 10}=6.90, p=0.025\right)$ was obtained. As depicted in Figure 4A and indicated by post hoc $t$-test $(p<0.05$, corrected for multiple comparisons according to Benjamini and Hochberg, 1995) rTMS at the right FEF leads to attenuated cross-correlation strength between right prefrontal and posterior recording sites whereas coupling between left prefrontal cortex and posterior sites is not significantly influenced by rTMS of the right FEF. There was also one stimulation unspecific effect obtained: The interaction between factors PREFRONTAL SITE and POSTERIOR HEMISPHERE was significant $\left(F_{1 / 10}=5.81, p=0.037\right.$; see Figure 4B). Post hoc $t$-test $(p<0.05$, corrected for multiple comparisons according to Benjamini and Hochberg, 1995) indicate that independent of rTMS left prefrontal site FC3 exhibited stronger coupling to right than left posterior sites. In general there was stronger coupling between left posterior sites and (right prefrontal site) FC4 compared to FC3. Of note is also that for recording site FC4 (electrode position closest to right FEF) there was no significant difference in coupling strength with left vs. right posterior sites. This is further evidence for the importance of the right FEF in top-down control of visual brain areas during directed attention.

In a directed attention paradigm Capotosto et al. (2009) could show that rTMS induced alterations of posterior alpha amplitude were correlated with behavioral effects of magnetic stimulation on visual attention. Therefore, here we also investigated associations between attenuated ipsilateral alpha amplitude after stimulation of the right FEF and increased response times compared to rTMS at the vertex. Alpha amplitude difference values between the vertex rTMS and the right FEF rTMS condition were correlated to respective difference values of response time in valid conditions (in which significant behavioral effects had been found; see above). This was done for parietal, parieto-occipital, and occipital electrodes left and right. rTMS induced alpha amplitude change at electrode site P8 was negatively correlated to response time change $(r=-0.603, p<0.05$; one-tailed, corrected for multiple comparisons according to Benjamini and Hochberg, 1995) when attention was directed to the right visual hemi-field. This 


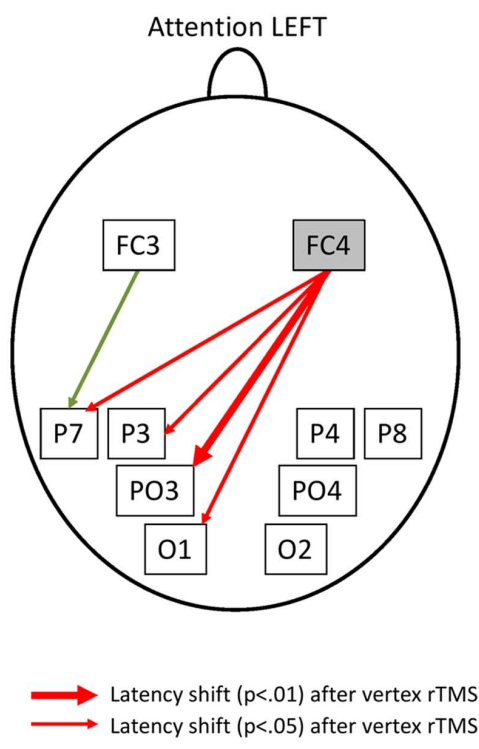

FIGURE 3 | Stable upper alpha latency shifts during directing of visuospatial attention. Arrows indicate consistent upper alpha latency shifts between recording sites. Note that prefrontal sites are always leading posterior sites during shifts of visuospatial attention. Red arrows indicate

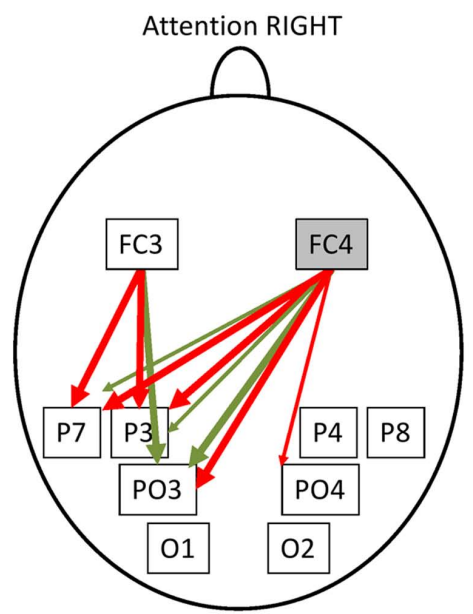

$\longrightarrow$ Latency shift $(p<.01)$ after right frontal rTMS $\longrightarrow$ Latency shift $(\mathrm{p}<.05)$ after right frontal rTMS

stable latency shifts after rTMS at the control stimulation site whereas green arrows indicate latency shifts after FEF rTMS. Top-down control from (right) prefrontal to posterior electrodes is strongly attenuated after stimulation of the right FEF. means that subjects showing a stronger decrease of ipsilateral (right hemispheric) alpha amplitude after rTMS at the right FEF compared to vertex also exhibited a larger increase in response times in valid trials when attention was shifted to the right visual hemi-field. Finally, the relation between rTMS induced changes in functional coupling between prefrontal and posterior sites and rTMS induced changes of response times was investigated. Difference values between fronto-posterior cross-correlation coefficients obtained after rTMS at the vertex and stimulation of the right FEF were correlated with response time difference values (vertexFEF stimulation) obtained from valid trials. Significant negative correlations were obtained for electrode pair FC4-P4 $(r=-0.67$, $p<0.05$; one-tailed, corrected for multiple comparisons according to Benjamini and Hochberg, 1995) when attention was directed to the left visual hemi-field, as well as for electrode pairs FC3-PO3 ( $r=-0.65, p<0.05$; one-tailed, corrected for multiple comparisons according to Benjamini and Hochberg, 1995) and FC4-P3 ( $r=-0.70, p<0.01$; one-tailed, corrected for multiple comparisons according to Benjamini and Hochberg, 1995) when the right visual hemi-filed was attended (see Figure 5).

\section{DISCUSSION}

By setting a transient virtual lesion at the right FEF it was investigated whether this cortical region was involved in top-down control of parietal and occipital attention-related lateralized EEG alpha activity and which role interregional functional coupling had during this top-down control process. The main findings were (i) a slowing of response times in valid trials, (ii) attenuation of lateralized upper alpha amplitude at posterior recording sites, (iii) decreased number of fronto-parietal electrode pairs showing significant and stable upper alpha latency shifts from prefrontal to posterior sites, (iv) decreased strength of maximal cross-correlation coefficients between right fronto-central and bilateral posterior electrode sites, and (v) significant association between rTMS induced response time delays and rTMS induced reduction of ipsilateral alpha amplitude and fronto-parietal crosscorrelation strength. These findings are well in line with recent evidence coming from a visual working memory task in which rTMS was applied over the right inferior frontal junction (Zanto et al., 2011). Zanto et al. (2011) found interregional phase synchronization at EEG alpha frequency between right prefrontal and posterior electrode sites reduced and so was performance in the task.

The behavioral effects of rTMS in the present study were rather moderate. An ANOVA on response times did only indicate faster response times for valid compared to invalid trials and the interaction with stimulation was only marginally significant. Only pairwise comparisons suggest that exclusively for valid trials response times are slower after rTMS to the right FEF than after rTMS to a control site. These moderate effects of right FEF stimulation on behavior in a cued attention task are in line with moderate behavioral results in the study by Taylor et al. (2007). Also Capotosto et al. (2009) reported stronger effects for rTMS at parietal sites than after stimulation of the right FEF. It is of note that previous research reports an increase in visual perception performance when the FEF is magnetically stimulated (Grosbras and Paus, 2002). However, in contrast to the current study Grosbras and Paus (2002) applied single TMS pulses shortly before presentation of a visual target. Slow rTMS as used in the current study might much more clearly lead to a sustained inhibition of the FEF rather than a very transient virtual lesion based on induction of neural noise. This could explain why here we rather find deceleration of response times than a speeding up after rTMS at the right FEF. Smith et al. (2009) used an on-line rTMS approach similar to Taylor et al. (2007) and 

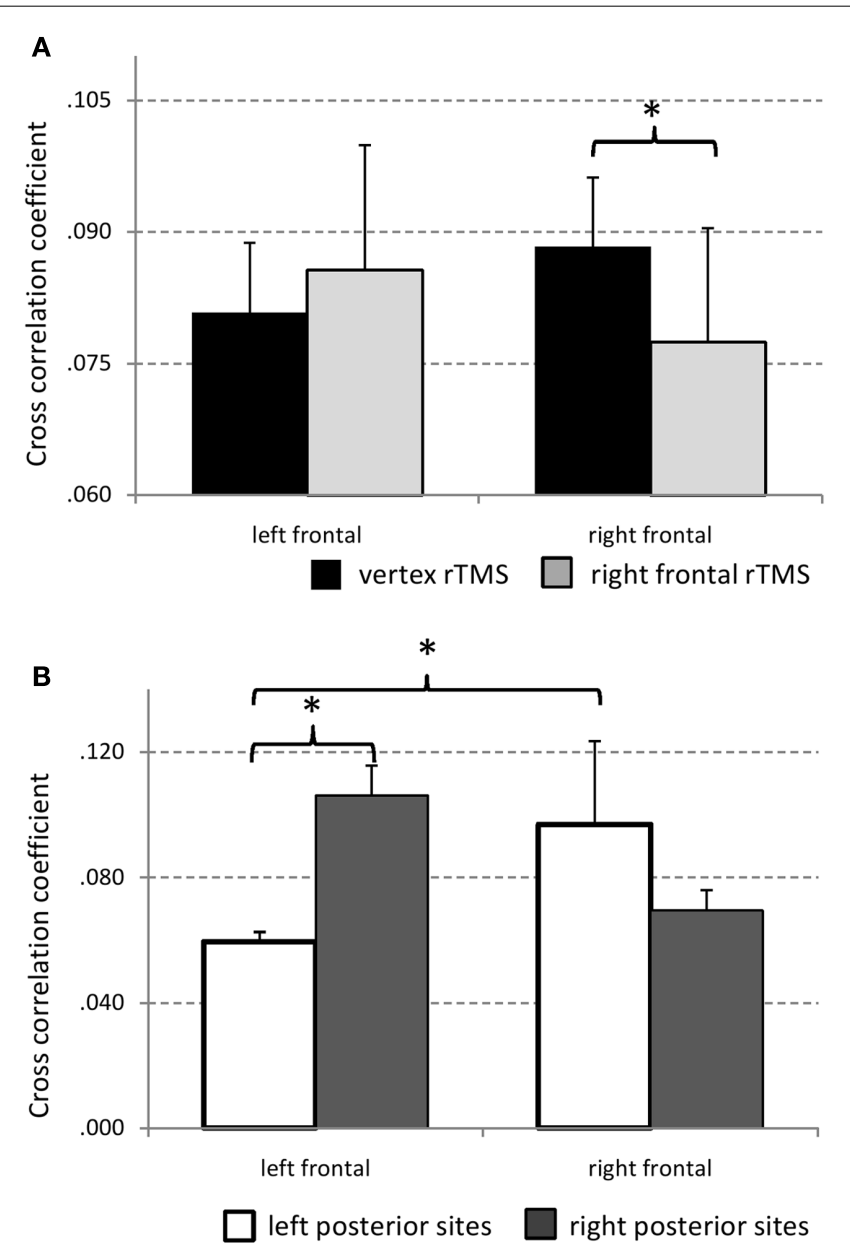

$* \mathrm{p}<.05$

FIGURE 4 | (A) Maximal cross-correlation coefficient as a function of hemisphere and stimulation condition. Averaged maxima of cross-correlation coefficient between recording site FC4 and all posterior electrodes of interest as well as FC3 and posterior recording sites are shown. After rTMS to the right FEF attenuated cross-correlation coefficients between FC4 (overlying the stimulation site) and posterior sites is obtained. (B) Hemispheric differences of cross-correlation strength between frontal and posterior sites. Note that in contrast to FC3 there is no significant difference found for coupling between right frontal and either left or right posterior sites. This suggests the right FEF to top-down control bilateral visual cortex.

Capotosto et al. (2009) in which in each single-trial a high frequency rTMS burst was delivered to the FEF. Smith et al. (2009) obtained faster response times to invalid trials in response to real compared to sham rTMS. In the present paper slower reaction times in response in valid trials after rTMS of the right FEF are reported. Although these findings seem contradictory, it must be noted that both effects, faster response times in invalid trials and slower reaction times in valid trials, reflect impairment of attentional control. When there is a failure of top-down control visual attention is not focused well on the cued visual hemi-field. Thus, in valid trials visual processing of a target might not be boosted by attention leading to slower response times. At the same time a weak focus of attention on the cued visual hemi-field, however, is of advantage in invalid trials since attention does not need to be reoriented, leading to faster response times after rTMS over FEF. It is not completely clear why Smith et al. (2009) found an effect only on invalid trials and here we only obtained significant effects in valid trials. The fact that Smith et al. (2009) used an auditory attention paradigm and that different stimulation protocols were used between the two studies might be the reason for this divergence.

Similar to Capotosto et al.'s (2009) findings, attenuated lateralized attention-related alpha amplitude was obtained at posterior sites after rTMS of the right FEF in this experiment. After stimulation of the control site a well-described pattern of upper alpha activity was elicited by shifting visuospatial attention: there was clearly higher alpha amplitude at sites ipsilateral to the attended location than contralateral to it. This is in line with recent literature on the role of EEG alpha oscillations in visual attention (Worden et al., 2000; Sauseng et al., 2005a; Thut et al., 2006; Rihs et al., 2007, 2009; Freunberger et al., 2008). These data support the view that high alpha amplitude reflects inhibition of information processing (Hummel et al., 2002; Jensen et al., 2002; Klimesch et al., 2007; Romei et al., 2008; Freunberger et al., 2009; Sauseng et al., 2009a,b; Jensen and Mazaheri, 2010). Increased ipsilateral alpha activity therefore indicates inhibitory biasing of visual perception leading to a processing advantage of attended locations in the visual field. This inhibitory mechanism seems to be controlled (at least in part) by the right FEF since the attention-related activation pattern was completely lacking after setting a virtual lesion at this brain region.

The top-down control of posterior inhibitory alpha amplitude by prefrontal cortex was already suggested by Sauseng et al. (2005b). In line with these findings, the current study showed also upper alpha latency shifts from prefrontal to posterior recording sites which are supposed to reflect inhibitory top-down control of higher visual brain areas. Notably, in the control condition when attention was shifted to the left there was significant top-down influence from right prefrontal site to only left (ipsilateral) posterior sites, thus, recording sites also exhibiting increased attentionrelated alpha amplitude. During shifts of attention toward the right visual hemi-field functional top-down coupling from left prefrontal but also from right prefrontal sites to posterior brain areas was obtained. This emphasizes the importance of the right FEF during control of visuospatial attention shifts to the left but interestingly also to the right. It is of note that all these effects of right prefrontal to posterior top-down functional coupling are strongly attenuated after rTMS delivered to the right FEF. This argues for necessity of the right FEF for attention-based top-down control of parietal and occipital upper alpha activity. However, it is remarkable, that despite a general reduction of fronto-parietal connectivity after rTMS at the right FEF there are significant stable latency shifts between electrode sites FC3 and P7 as well as PO3 which are not found in the control condition. This indicates that a lesion to the right FEF might lead to compensatory top-down control of posterior cortical areas by the left FEF.

Perhaps one of the most important findings in the present study is that of significant correlations between the impact of right FEF rTMS on the strength of cross-correlations between prefrontal and parietal sites and the impact on response times in valid trials. A 

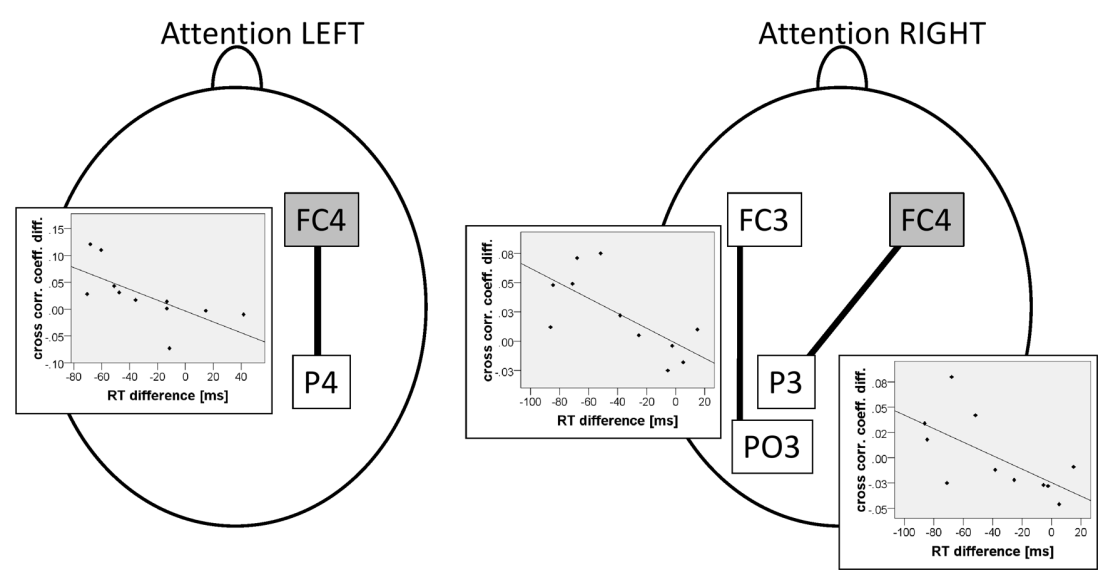

FIGURE 5 | Correlations between rTMS dependent interregional coupling strength and response times. Subjects exhibiting a stronger attenuation of cross-correlation coefficients after stimulation of the right FEF compared to vertex rTMS show larger response time increase. Note that in this effect there are always posterior sites involved that are located contralateral to the attended visual hemi-field. larger response time delay was associated with greater attenuation of fronto-parietal coupling. Interestingly, only electrode pairs between frontal and contralateral posterior sites showed significant association between behavioral effects of rTMS and altered cross-correlation strength. This suggests a highly specific function of fronto-parietal long-range alpha coupling in visual attention.

We did not only obtain significant correlations between behavior and interregional connectivity but also with local alpha amplitude. Well in line with Capotosto et al.'s (2009) results attenuated ipsilateral alpha amplitude was associated with prolonged response times. However, this effect was only apparent for one right parietal electrode and was absent at those sites showing the strongest effects of rTMS on ipsilateral alpha amplitude (left posterior sites).

Although overall behavioral effects have been weak in this study (see Discussion above), the correlation between altered EEG patterns and response time delays is evidence for a causal role of fronto-parietal top-down alpha activity controlling visuospatial attention.

Corbetta and Shulman (2002) discuss the relevance of a frontoparietal right hemispheric attention network in humans. Whereas our results point to the importance and hemispheric dominance of the right FEF compared to the left frontal cortex, it is interesting that mainly left-hemispheric posterior recording sites show strong attention-related effects in the present study. There are stronger local alpha amplitude as well as interregional coupling effects found for left posterior than right posterior sites. However, it must not be missed that we interpret alpha activity as neural mechanism of inhibition (Klimesch et al., 2007) and top-down inhibitory control (Sauseng et al., 2005b). Since active attention control processes are implemented in the right parietal cortex (Corbetta and Shulman, 2002; Capotosto et al., 2009) these brain areas might be under less inhibitory control from the FEF than left-hemispheric visual cortex seems to be.

An important issue which needs to be addressed, however, is whether rTMS induced effects on local alpha amplitude and interregional alpha coupling in this study are independent from each other or not. Theoretically cross-correlations (in particular latency shifts) should be independent from amplitude of the underlying signals. But it can be argued that phase of a signal (and cross-correlation coefficients seem to be most sensitive to stable phase relationship of two signals) is most reliably estimated when the underlying signal is of large amplitude. To control for that we correlated alpha amplitude from posterior recording sites with maximal cross-correlation coefficients for each site/electrode pair. From these 64 correlations 10 were significant (without correction for multiple testing). These correlations were only found involving posterior electrode sites $\mathrm{P} 4$ and $\mathrm{P} 8$ and were unspecific on whether attention was directed to the left or right visual field. We did the same correlations between posterior alpha amplitude and crosscorrelation latency shifts and found only 3 out of 64 correlations to be significant. With two of these being positive and one being negative this pattern however seemed to be completely random. This indicates that results on local alpha amplitude and interregional connectivity are vastly independent from each other in this study.

\section{CONCLUSION}

Based on the findings of this study it can be suggested that the right FEF is strongly involved in the control of posterior attentionrelated alpha activity. The mechanism by which this control is achieved seems to be interregional synchronization. The results from the present study highlight the importance of fronto-parietal connectivity at alpha frequency for top-down control of visual processing. Of course, the current findings also indicate that control of visuospatial attention might only partly rely on top-down influence of the right FEF on posterior brain areas. There might be other (top-down) mechanisms and other brain structures strongly involved in the process of directed visuospatial attention.

\section{ACKNOWLEDGMENTS}

This research was supported by the Austrian Academy of Science (Paul Sauseng was an APART fellow of the Austrian Academy of Science at the University of Salzburg) and the Austrian Science Fund FWF (P 22084-B18). 


\section{REFERENCES}

Benjamini, Y., and Hochberg, Y. (1995). Controlling the false discovery rate: a practical and powerful approach to multiple testing. J. R. Stat. Soc. B 57, 289-300.

Bressler, S. L., Tang, W., Sylvester, C. M., Shulman, G. L., and Corbetta, M. (2008). Top-down control of human visual cortex by frontal and parietal cortex in anticipatory visual spatial attention. J. Neurosci. 28, 10056-10061.

Capotosto, P., Babiloni, C., Romani, G. L., and Corbetta, M. (2009). Frontoparietal cortex controls spatial attention through modulation of anticipatory alpha rhythms. J. Neurosci. 29, 5863-5872.

Chen, R., Classen, J., Gerloff, C., Celnik, P., Wassermann, E. M., Hallett, M., and Cohen, L. G. (1997). Depression of motor cortex excitability by low-frequency transcranial magnetic stimulation. Neurology 48, 1398-1403.

Corbetta, M., and Shulman, G. L. (2002). Control of goal-directed and stimulus-driven attention in the brain. Nat. Rev. Neurosci. 3, 201-215.

Di Russo, F., Martinez, A., and Hillyard, S. A. (2003). Source analysis of event-related cortical activity during visuo-spatial attention. Cereb. Cortex 13, 486-499.

Freunberger, R., Fellinger, R., Sauseng, P., Gruber, W., and Klimesch, W. (2009). Dissociation between phaselocked and nonphase-locked alpha oscillations in a working memory task. Hum. Brain Mapp. 30, 3417-3425.

Freunberger, R., Höller, Y., Griesmayr, B., Gruber, W., Sauseng, P., and Klimesch, W. (2008). Functional similarities between the P1 component and alpha oscillations. Eur. J. Neurosci. 27, 2330-2340.

Green, J. J., and McDonald, J. J. (2008). Electrical neuroimaging reveals timing of attentional control activity in human brain. PLoS Biol. 6, e81. doi:10.1371/journal.pbio.0060081

Grosbras, M.-H., and Paus, T. (2002). Transcranial magnetic stimulation of the human frontal eye field: effects on visual perception and attention. J. Cogn. Neurosci. 14, 1109-1120.

Hillyard, S. A., and Anllo-Vento, L. (1998). Event-related brain potentials in the study of visual selective attention. Proc. Natl. Acad. Sci. U.S.A. 95, 781-787.

Hummel, F., Andres, F., Altenmüller, E., Dichgans, J., and Gerloff, C.
(2002). Inhibitory control of acquired motor programmes in the human brain. Brain 125, 404-420.

Jensen, O., Gelfand, J., Kounios, J., and Lisman, J. E. (2002). Oscillations in the alpha band $(9-12 \mathrm{~Hz})$ increase with memory load during retention in a short-term memory task. Cereb. Cortex 12, 877-882.

Jensen, O., and Mazaheri, A. (2010). Shaping functional architecture by oscillatory alpha activity: gating by inhibition. Front. Hum. Neurosci. 4:186. doi:10.3389/fnhum.2010.00186

Klimesch, W., Sauseng, P., and Hanslmayr, S. (2007). EEG alpha oscillations: the inhibition-timing hypothesis. Brain Res. Rev. 53, 63-88.

Moore, T., and Armstrong, K. M. (2003). Selective gating of visual signals by microstimulation of frontal cortex. Nature 421, 370-373.

Moore, T., and Fallah, M. (2001). Control of eye movements and spatial attention. Proc. Natl. Acad. Sci. U.S.A. 98, 1273-1276.

O'Shea, J., Muggleton, N. G., Cowey, A., and Walsh, V. (2004). Timing of target discrimination in human frontal eye fields. J. Cogn. Neurosci. 16, 1060-1067.

Rihs, T. A., Michel, C. M., and Thut, G. (2007). Mechanisms of selective inhibition in visual spatial attention are indexed by $\alpha$-band EEG synchronization. Eur. J. Neurosci. 25, 603-610.

Rihs, T. A., Michel, C. M., and Thut, G. (2009). A bias for posterior $\alpha$-band power suppression versus enhancement during shifting versus maintenance of spatial attention. Neuroimage 44, 190-199.

Romei, V., Brodbeck, V., Michel, C., Amedi, A., Pascual-Leone, A., and Thut, G. (2008). Spontaneous fluctuations in posterior $\alpha$-band EEG activity reflect variability in excitability of human visual areas. Cereb. Cortex 18, 2010-2018.

Romei, V., Gross, J., and Thut, G. (2010). On the role of prestimulus alpha rhythms over occipito-parietal areas in visual input regulation: correlation or causation? J. Neurosci. 30, 8692-8697.

Rossi, S., Hallett, M., Rossini, P. M., and Pascual-Leone, A. (2009). Safety, ethical considerations, and application guidelines for the use of transcranial magnetic stimulation in clinical practice and research. Clin. Neurophysiol. 120 , 2008-2039.

Rossini, P. M., Barker, A. T., Berardelli, A., Caramia, M. D., Caruso, G., Cracco, R. Q., Dimitrijevic, M. R., Hallett, M., Katayama, Y., Lucking, C. H., Maertens De Noordhout, A. L., Marsden, C. D., Murray, N. M. F., Rothwell, J. C., Swash, M., and Tomberg, C. (1994). Non-invasive electrical and magnetic stimulation of the brain, spinal cord and roots: basic principles and procedures for routine clinical application. Report of an IFCN committee. Electroencephalogr. Clin. Neurophysiol. 91, 79-92.

Sauseng, P., Klimesch, W., Doppelmayr, M., Hanslmayr, S., Schabus, M., and Gruber, W. R. (2004). Theta coupling in the human electroencephalogram during a working memory task. Neurosci. Lett. 354 123-126.

Sauseng, P., Klimesch, W., Heise, K. F., Gruber, W. R., Holz, E., Karim, A. A., Glennon, M., Gerloff, C., Birbaumer, N., and Hummel, F. C. (2009a). Brain oscillatory substrates of visual short-term memory capacity. Curr. Biol. 19, 1846-1852.

Sauseng, P., Klimesch, W., Gerloff, C., and Hummel, F. C. (2009b). Spontaneous locally restricted EEG alpha activity determines cortical excitability in the motor cortex. Neuropsychologia 47, 284-288.

Sauseng, P., Klimesch, W., Stadler, W., Schabus, M., Doppelmayr, M. Hanslmayr, S., Gruber, W. R., and Birbaumer, N. (2005a). A shift of visual spatial attention is selectively associated with human EEG alpha activity. Eur. J. Neurosci. 22, 2917-2926.

Sauseng, P., Klimesch, W., Doppelmayr, M., Pecherstorfer, T., Freunberger, R., and Hanslmayr, S. (2005b). EEG alpha synchronization and functional coupling during top-down processing in a working memory task. Hum. Brain Mapp. 26, 148-155.

Schack, B., Weiss, S., and Rappelsberger, P. (2003). Cerebral information transfer during word processing: where and when does it occur and how fast is it? Hum. Brain Mapp. 19, 18-36

Smith, D. T., Jackson, S. R., and Rorden C. (2009). Repetitive transcranial magnetic stimulation over frontal eye fields disrupts visually cued auditory attention. Brain Stimul. 2 , 81-87.
Taylor, P. C. J., Nobre, A. C., and Rushworth, M. F. S. (2007). FEF TMS affects visual cortical activity. Cereb. Cortex 17, 391-399.

Thut, G., Nietzel, A., Brandt, S. A., and Pascual-Leone, A. (2006). $\alpha$-Band electroencephalographic activity over occipital cortex indexes visuospatial attention bias and predicts visual target detection. J. Neurosci. 26, 9494-9502.

Wassermann, E. M. (1998). Risk and safety of repetitive transcranial magnetic stimulation: report and suggested guidelines from the international workshop on the safety of repetitive transcranial magnetic stimulation, June 5-7, 1996. Electroencephalogr. Clin. Neurophysiol. 108, 1-16.

Worden, M. S., Foxe, J. J., Wang, N., and Simpson, G. V. (2000). Anticipatory biasing of visuospatial attention indexed by retinotopically specific alpha-band electroencephalography increases over occipital cortex. J. Neurosci. 20, RC63.

Zanto, T. P., Rubens, M. T., Thangavel, A., and Gazzaley, A. (2011) Causal role of the prefrontal cortex in top-down modulation of visual processing and working memory. Nat. Neurosci. 14, 656-661.

Conflict of Interest Statement: The authors declare that the research was conducted in the absence of any commercial or financial relationships that could be construed as a potential conflict of interest.

Received: 28 February 2011; accepted: 03 September 2011; published online: 05 October 2011.

Citation: Sauseng P, Feldheim JF, Freunberger $R$ and Hummel FC (2011) Right prefrontal TMS disrupts interregional anticipatory EEG alpha activity during shifting of visuospatial attention. Front. Psychology 2:241. doi: 10.3389/fpsyg.2011.00241

This article was submitted to Frontiers in Perception Science, a specialty of Frontiers in Psychology.

Copyright (C) 2011 Sauseng, Feldheim, Freunberger and Hummel. This is an open-access article subject to a nonexclusive license between the authors and Frontiers Media SA, which permits use, distribution and reproduction in other forums, provided the original authors and source are credited and other Frontiers conditions are complied with. 